\title{
Total neoadjuvant therapy in patients with locally advanced rectal cancer: A tertiary medical center experience
}

\author{
ZIAD EL HUSSEINI, YOLLA HAIBE, YOUSSEF BOUFERRAA, MALEK KREIDIEH, MONITA AL DARAZI, \\ DEBORAH MUKHERJI, SALLY TEMRAZ, MAYA CHARAFEDDINE and ALI SHAMSEDDINE
}

\author{
Department of Internal Medicine, Division of Hematology/Oncology, American University \\ of Beirut Medical Center, Riad El Solh, Beirut 1107 2020, Lebanon
}

Received April 19, 2021; Accepted June 3, 2021

DOI: $10.3892 /$ mco.2021.2382

\begin{abstract}
The current standard of care for locally advanced rectal cancer (LARC) includes preoperative chemoradiation, followed by total mesorectal excision and adjuvant chemotherapy. This multimodality treatment improves local control but is associated with low compliance rates without clear beneficial effects on overall survival (OS) and distant metastasis. In this retrospective study, the charts of patients diagnosed with cT3/4 or cT2-node-positive rectal cancer between January 2011 and June 2019 were reviewed. The chemoradiation therapy (CRT) group received a long course of CRT with capecitabine followed by surgery and adjuvant chemotherapy. The total neoadjuvant therapy (TNT) group received 6 cycles mFOLFOX and a short course of radiation therapy followed by surgery. A total of 81 patients were included, among which 55 (67.9\%) received CRT and 26 (32.1\%) received TNT. In the CRT group, 15 (27.3\%) patients achieved pathologic complete response (pCR) compared with 10 (38.5\%) in the TNT group $(\mathrm{P}=0.22)$. A total of 19 (35.8\%) cases in the CRT group downstaged to pT0N0 or pT1N0 compared with $11(42.3 \%)$ in the TNT group $(\mathrm{P}=0.33)$. The 2-year disease-free survival (DFS) rate was $81.0 \%$ in the TNT group and $84.0 \%$ in the CRT group $(\mathrm{P}=0.15)$. Out of 55 patients in the CRT group, 30 patients received adjuvant chemotherapy, 22 (40.0\% of CRT cases) of which completed a full course. All 26 patients in the TNT group received neoadjuvant chemotherapy, where $22(84.6 \%)$ patients took a full course $(\mathrm{P}<0.001)$. In conclusion, the present study revealed that patients treated with TNT were more compliant to chemotherapy than those treated with CRT. A numerically higher $\mathrm{pCR}$ rate, and nodal and tumor downstaging were noted in the TNT group without significance. No
\end{abstract}

Correspondence to: Dr Ali Shamseddine, Department of Internal Medicine, Division of Hematology/Oncology, American University of Beirut Medical Center, Abdul Aziz Street, Riad El Solh, Beirut 1107 2020, Lebanon

E-mail: as04@aub.edu.lb

Key words: rectal neoplasm, total neoadjuvant therapy, disease-free survival, compliance, chemotherapy, oncology difference was noted in the 2-year DFS. Longer follow-up is required.

\section{Introduction}

For the past 3 decades, we have been witnessing dynamic changes in the treatment of locally advanced rectal cancer (LARC) from single modality treatment using surgery alone to multimodality treatment including radiation, chemotherapy and total mesorectal excision (TME). These changes led to an improvement in outcome for stage II and III rectal cancer (1).

TME has made a revolution in the management of rectal cancer as it has shown a decrease in local recurrence from $30-50 \%$ to $\sim 5 \%$ and allowed the patients to have sphincter preserving surgery (2). The use of adjuvant chemotherapy is advisable, but it is still controversial as studies have not shown an improvement in distant recurrence, overall survival (OS) and disease-free survival (DFS) (3). Compliance to adjuvant treatment has been a major concern in our daily practice and this may have affected the survival outcome. In fact, some studies have shown that a 4-week delay in post-operative treatment correlated with a decrease in OS of 14\% (4-6).

In terms of sequencing, German trial, along with similar trials, showed that preoperative chemo-radiation therapy combined with TME have shown better compliance, less toxicity and less local pelvic recurrence rate as compared to post-operative radiation treatment. Moreover, they detected an increase in pathologic complete response (pCR) rate with chemo-radiation therapy that correlates with better oncologic outcomes and may play an important role in organ preservation strategy (7-11).

Currently, the accepted standard of care for treatment of LARC is neoadjuvant chemo-radiation followed by TME and adjuvant chemotherapy. Despite the improvement in local control resulting from the association of different treatment regimens, the death rate from rectal cancer from distant metastasis is still elevated compared to local failure (12). While this multimodality approach improved local control, no impact was noted on the OS, with distant metastasis remaining the most concerning issue with a cumulative incidence of $30 \%$ in 10 years and with an overall DFS of $68 \%$ at 10 years (13). Therefore, better systemic control is needed. This could be 
potentially achieved by focusing more on micrometastasis early in the course of treatment, hence delivering chemotherapy in pre-operative setting. This regimen, known as total neoadjuvant therapy (TNT), consists of delivering chemotherapy and radiation therapy in neoadjuvant setting. TNT was proposed to treat micrometastasis, increase pCR rate and increase compliance to treatment.

The idea of TNT was developed in the RAPIDO trial that was first launched in 2011 (14). It showed impressive results that were presented at ASCO 2020 and that may lead to a change in standard of care with TNT regimen. In this trial, TNT was associated with lower disease-related treatment failure, distant metastasis rate and doubling of pathologic complete response ( $\mathrm{pCR}$ ) rate compared to standard of care (15).

In this retrospective study, we assess the difference in pCR rates, compliance to chemotherapy, tumor downstaging and DFS between TNT and chemoradiation therapy (CRT) for LARC at the American University of Beirut Medical Center (AUBMC). We present this article in accordance with the STROBE reporting checklist.

\section{Materials and methods}

Study design and patient selection. This study is a retrospective chart review of patients diagnosed with LARC at the American University of Beirut Medical Center between January 1st, 2011, and June 1st, 2019. Patients $\geq 18$ years old and with cT3/4 or cT2-node-positive were included. In total, 81 patients were included in the study and patients' demographics, treatment course and clinical outcomes were recorded. Charlson Comorbidity Index (CCI) was also calculated for every patient. The study was conducted in accordance with the Declaration of Helsinki (as revised in 2013). The study was approved via expedited review by the local Institutional Review Board (IRB) committee at AUBMC IRB ID: BIO-2019-0024.

Study objectives. The primary endpoint is the pathologic complete response (pCR) rate between the two different treatment modalities. pCR was defined as the absence of any viable tumor cell within the tumor bed in the setting of chemotherapy effect (16). The secondary endpoints are the compliance to chemotherapy, tumor downstaging and DFS.

Treatment modalities. The change in treatment modalities between patients is due to advancement in the field. Patients included were divided into either TNT or CRT groups. The TNT is defined as short course radiotherapy ( $5 \times 5 \mathrm{~Gy}$ ) before or after 6 cycles of mFOLFOX (oxaliplatin, fluorouracil and folinic acid) followed by TME. TME was performed 4 weeks after neoadjuvant therapy in the TNT group. The total duration of treatment between was 16-18 weeks. The CRT is defined as concurrent long course radiotherapy (45 Gy divided in 25 fractions over 5 weeks) and capecitabine followed by TME and adjuvant chemotherapy. TME involves the removal of the rectum together with surrounding mesorectum (lymphovascular fatty tissue) through a precise dissection along the pelvic visceral fascia (17). Treatment modalities were standardized between patients within the same treatment group.
Table I. Patient demographics.

\begin{tabular}{|c|c|c|}
\hline \multirow[b]{2}{*}{ Variables } & \multicolumn{2}{|c|}{ Treatment modality } \\
\hline & TNT & CRT \\
\hline Median age, years (range) & $51(25-75)$ & $60(34-85)$ \\
\hline \multicolumn{3}{|l|}{ Sex, n $(\%)$} \\
\hline Male & $16(61.5)$ & $32(58.2)$ \\
\hline Female & $10(38.5)$ & $23(41.8)$ \\
\hline \multicolumn{3}{|l|}{ Tumor differentiation, n (\%) } \\
\hline Well & $4(15.3)$ & $6(10.9)$ \\
\hline Moderate & $15(57.7)$ & $39(70.9)$ \\
\hline Poor & $6(23.1)$ & $1(1.8)$ \\
\hline Not documented & $1(3.9)$ & $9(16.4)$ \\
\hline \multicolumn{3}{|l|}{ Clinical stage, n (\%) } \\
\hline $\mathrm{T} 2 \mathrm{~N} 1$ & $0(0.0)$ & $1(1.8)$ \\
\hline T3N0 & $2(7.7)$ & $9(16.4)$ \\
\hline T3N1 & $9(34.6)$ & $34(61.8)$ \\
\hline $\mathrm{T} 3 \mathrm{~N} 2$ & $9(34.6)$ & $6(10.9)$ \\
\hline $\mathrm{T} 3 \mathrm{Nx}$ & $1(3.9)$ & $3(5.5)$ \\
\hline T4N0 & $0(0.0)$ & $1(1.8)$ \\
\hline T4N1 & $2(7.7)$ & $1(1.8)$ \\
\hline $\mathrm{T} 4 \mathrm{~N} 2$ & $3(11.5)$ & $0(0.0)$ \\
\hline T4Nx & $0(0.0)$ & $0(0.0)$ \\
\hline
\end{tabular}

CRT, chemoradiation therapy; TNT, total neoadjuvant therapy.

Statistical analysis. A biomedical statistician performed the statistical review of the study. Continuous variables were summarized by their median, mean and range. Categorical variables were described by counts and relative frequencies. Crosstabulations in the form of $2 \times 2$ tables were plotted to compared and detect differences between the two groups in outcome, downstaging and pathology. DFS curve was plotted using the Kaplan-Meier curve, the log rank was used to check for significant difference between the studied groups. DFS time was defined as the time from initial diagnosis to disease progression or the end of follow-up (censored observations who did not reach the progression event). A value of $\mathrm{P}<0.05$ was considered significant in all analyses. All statistical analysis was performed using the SPSS v.25.0 statistical package.

\section{Results}

Patient characteristics and distribution. Of the 81 patients diagnosed with LARC, 48 (59.3\%) were males and 33 (40.7\%) were females with the average age 59 (34-81) and 55 (25-85) years, respectively. Patients were distributed into two treatment groups; 26 patients received TNT and 55 received CRT with the average age 51 and 60 years, respectively (Table I). The median follow-up periods for the TNT and CRT groups were 22.7 and 47.8 months, respectively. The mean CCI was 3.96 in the CRT group and 3.23 in the TNT group $(\mathrm{P}<0.05)$.

Pathologic and survival outcomes. Of the 26 patients that received TNT, 10 (38.5\%) patients had a pCR, while of the 
Table II. Treatment outcome.

\begin{tabular}{lcc}
\hline & \multicolumn{2}{c}{ Treatment modality } \\
\cline { 2 - 3 } Variables & TNT & CRT \\
\hline Pathologic response & & \\
Complete response (TRG 0) & $10(38.5)$ & $15(27.3)$ \\
Near complete response (TRG 1) & $3(11.5)$ & $5(9.1)$ \\
Other response (TRG 2 and 3) & $13(50.0)$ & $35(63.6)$ \\
Pathologic staging & & \\
ypT0N0 & $10(38.5)$ & $15(27.3)$ \\
ypTisN0 & $0(0.0)$ & $1(1.8)$ \\
ypT1N0 & $1(3.9)$ & $3(5.5)$ \\
ypT2N0 & $2(7.7)$ & $3(5.5)$ \\
ypT3N0 & $6(23.1)$ & $16(29.1)$ \\
ypT1N1 & $2(7.7)$ & $0(0.0)$ \\
ypT2N1 & $0(0.0)$ & $5(9.1)$ \\
ypT3N1 & $1(3.9)$ & $6(10.9)$ \\
ypT3N2 & $1(3.9)$ & $3(5.5)$ \\
ypT4N1 & $1(3.9)$ & $1(1.8)$ \\
ypT4N2 & $2(7.7)$ & $1(1.8)$ \\
ypTxN1 & $0(0.0)$ & $1(1.8)$ \\
\hline
\end{tabular}

CRT, chemoradiation therapy; TNT, total neoadjuvant therapy; TRG, tumor regression grade.

55 patients that received CRT, 15 (27.3\%) patients had a pCR $(\mathrm{P}=0.22)$. On the other hand, 7 (26.9\%) patients from the TNT group and 17 (30.9\%) patients from the CRT group had a pathologic node-positive $(\mathrm{P}=0.46)$. Moreover, downstaging to pT0N0 and pT1N0 was achieved in $11(42.3 \%)$ and $19(35.8 \%)$ patients in the TNT and CRT groups, respectively $(\mathrm{P}=0.33)$ (Table II). The 2-year DFS rate was 81 and $84 \%$ in the TNT and CRT groups, respectively $(\mathrm{P}=0.15)$ (Fig. 1).

Compliance to chemotherapy. Of the 55 patients that received CRT, 30 (54.5\%) patients received any number of cycles of adjuvant chemotherapy and 22 (40\%) of which received a full course of chemotherapy. All of the 26 patients in the TNT group received neoadjuvant chemotherapy with $22(84.6 \%)$ of which receiving a full course $(\mathrm{P}<0.01)$. None of CRT patients and 1 patient from the TNT group had a dose reduction.

\section{Discussion}

Clinical outcomes in patients with LARC have improved markedly especially significant decrease in local failure with the development in treatment regimens. Sauer et al (13) proved in a randomized trial that neoadjuvant chemoradiation was significantly superior in local control in comparison to adjuvant chemoradiation, but with no difference in OS or DFS. Then, in the phase III randomized trials, EORTC22921 and I-CNR-RT, the addition of adjuvant fluorouracil and folinic acid to preoperative chemoradiation did not improve OS and DFS in comparison to regular surveillance post-surgery $(18,19)$. Moreover, the PETACC-6 phase III trial compared neoadjuvant chemoradiation with capecitabine followed by 6 cycles of adjuvant capecitabine with or without oxaliplatin, before and after surgery. This trial also showed that the addition of oxaliplatin to capecitabine did not improve OS and DFS (20). Finally, in the German CAO/ARO/AIO-04 phase III trial, the addition of oxaliplatin to preoperative chemoradiation and adjuvant fluorouracil and folinic acid, led to a significant improvement in DFS and OS, even though the addition of oxaliplatin to capecitabine-based chemoradiation is not the standard of care (21). Furthermore, the compliance rates to adjuvant chemotherapy were low. In a multicenter retrospective review, only $44.1 \%$ received adjuvant chemotherapy after neoadjuvant chemoradiation and rectal surgery and, of those, only $56 \%$ were compliant to treatment (22). In comparison, in our cohort, $54.5 \%$ of the patients in the CRT group received adjuvant chemotherapy and $40 \%$ of which were compliant to treatment. While on the other hand, $100 \%$ of the patients in the TNT group received neoadjuvant chemotherapy and $84 \%$ were compliant to treatment $(\mathrm{P}<0.01)$.

Furthermore, in a phase II trial, Marco et al (23) showed that giving mFOLFOX6 after chemoradiation and before surgery was associated with better compliance and DFS rates. Indeed, in another phase II trial, delaying the surgery by giving up to 6 cycles of mFOLFOX after chemoradiation was associated with superior $\mathrm{pCR}$ rates in comparison to patients not receiving neoadjuvant chemotherapy (24). Similar to the findings in previous studies, the main causes of noncompliance in our patients were post-operative complications, drug-related toxicities and patients' preferences.

In a randomized trial, Ngan et al (25) compared the local recurrence rates between short-course radiotherapy (25 Gy in 5 fractions) and long-course chemoradiation (50.4 Gy in 28 fractions). No difference was noted between the 3-year local recurrence rates, OS, distant recurrence rate or late toxicity, all in favor of considering short-course radiotherapy to decrease the treatment time without any change in clinical outcomes (25).

Moreover, the Stockholm III trial compared by randomization short-course radiation immediately before surgery, short-course radiation with delayed surgery and long-course radiotherapy (50 Gy in 25 fractions) with delayed surgery. It showed that postoperative complications were significantly less in the group receiving short-course radiotherapy with delayed surgery, making this regimen practical (26). On the other hand, the Polish II randomized trial showed no difference, after 8 years, between short-course radiotherapy followed by neoadjuvant chemotherapy and upfront chemoradiotherapy in OS or DFS (27).

In an effort to truly assess the potential of neoadjuvant chemotherapy, Fokas et al (28) compared, in a randomized phase II trial, neoadjuvant chemotherapy given before or after chemoradiation followed by surgery. Chemoradiation followed by neoadjuvant chemotherapy and surgery was associated with superior $\mathrm{pCR}$ rates and better compliance with chemoradiation but worse compliance with chemotherapy (28). Most recently, in a randomized trial, short-course radiotherapy followed by neoadjuvant chemotherapy then by TME was compared to long-course radiotherapy followed by TME and optional adjuvant chemotherapy. The RAPIDO trial showed that the TNT treatment modality was significantly superior in pCR 


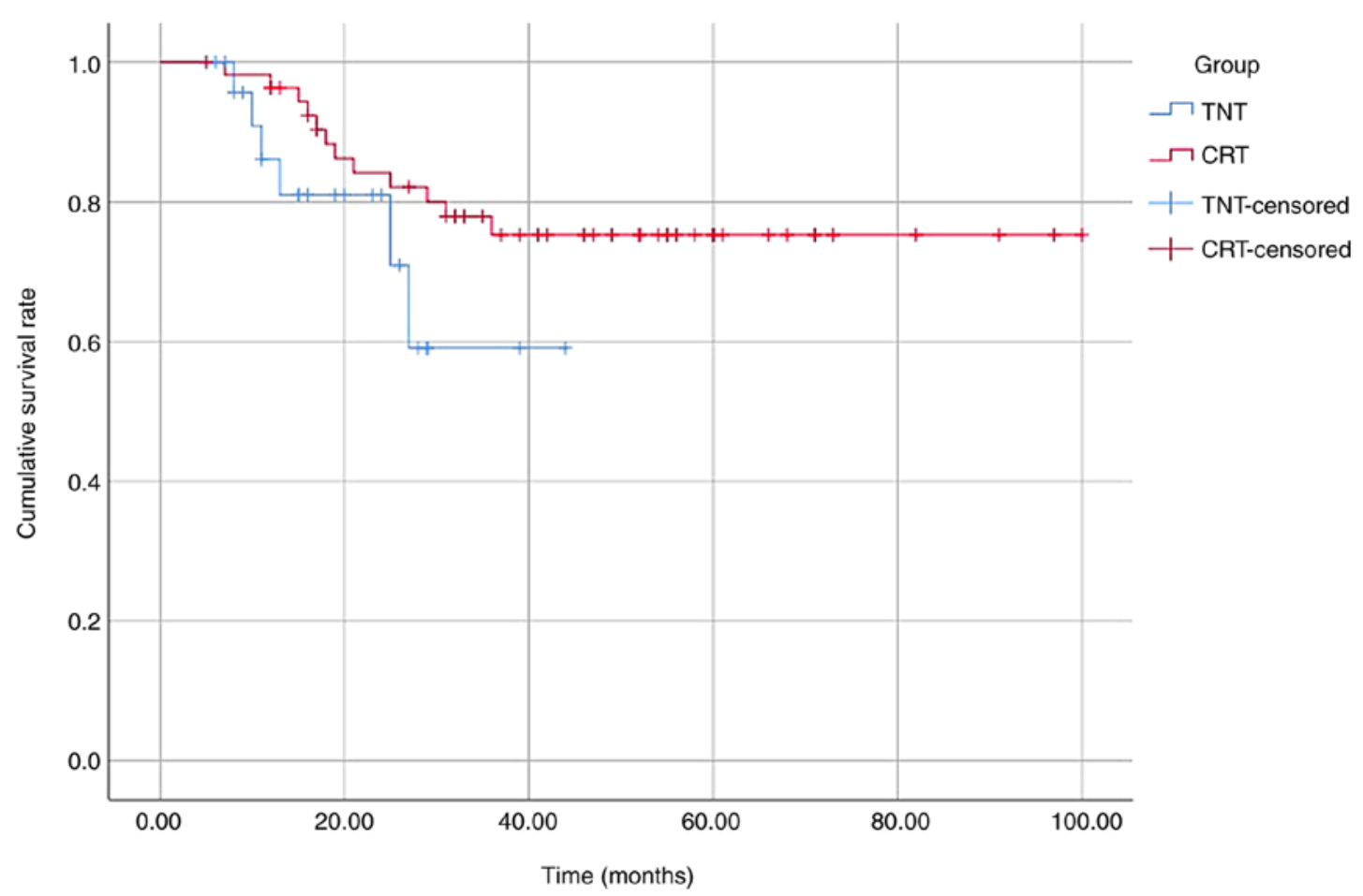

Figure 1. Two-year DFS in the TNT and CRT groups. The 2-year DFS rate was 81 and $84 \%$ in the TNT and CRT groups, respectively (P=0.15). DFS, disease-free survival; CRT, chemoradiation therapy; TNT, total neoadjuvant therapy.

rate (28 vs. $14 \%$ ) and had a $7 \%$ decrease in disease-related treatment failure (14). In our cohort, the pCR rate was numerically higher for the TNT group without statistical significance, which can be due to the small sample size. On the other hand, in a systemic review comparing the two treatment modalities, the pooled pCR rates were found to be 32.4 and $22.3 \%$, while in our cohort the rates were 38.5 and $27.3 \%$ in the TNT and CRT groups, respectively (29). Moreover, the difference in the 2 -years DFS rates between the two modalities was not significant, while only one study showed an improved DFS in the TNT group (29). In fact, the RAPIDO trial showed that the 3 -year OS rate was the same in both groups (14). Furthermore, our data showed that the TNT group had a numerically higher rate of tumor downstaging than the CRT group but without statistical significance. Chapman et al (30) compared the neoadjuvant rectal score between the two treatment modalities and showed that TNT is superior to the standard CRT in tumor downstaging between clinical and pathologic stage.

Furthermore, organ preservation has been advocated with the elevated rates of complete response reached with TNT. Patients in the OPRA trial were randomized into neoadjuvant chemotherapy before or after chemoradiation, and then patients with complete or near-complete response were offered watchful waiting. It showed that patients receiving upfront chemoradiation followed by neoadjuvant chemotherapy resulted in a significantly superior rates of organ preservation (31).

Currently, a clinical trial is the assessing the efficacy and toxicity of short-course radiation concurrently with 5-fluorouracil infusion for the treatment of LARC (NCT04370418) (32). Moreover, Zhang et al (33) are evaluating the efficacy and safety of dose escalation of short-course radiotherapy, from 25 to 40 Gy in 5 fractions, followed by chemotherapy and surgery. Additionally, immune checkpoint inhibitors have been gaining a great deal of attention lately. The VOLTAGE trial is assessing nivolumab (anti-programmed death-1) monotherapy after chemoradiation followed by surgery in LARC patients. The preliminary results showed a promising 30 and $60 \%$ pCR rates in microsatellite stable and unstable patients, respectively (34). Also, the DUREC trial is assessing the addition of durvalumab (anti-programmed death-ligand 1) to induction chemotherapy and short-course radiotherapy or long-course chemoradiation followed by surgery (35). Moreover, in a phase II trial, avelumab (anti-programmed death-ligand 1) was combined with neoadjuvant mFOLFOX after short-course radiotherapy followed by surgery. The preliminary results showed a promising $25 \%$ pCR rate and $50 \%$ major response rate (36).

There are several limitations facing this study. First, this is a retrospective chart review with a small sample size, therefore more patients are needed with extended follow up periods to compare 5-year DFS and OS. In addition, there is some inhomogeneity within the TNT group as some patients received chemotherapy before radiotherapy unlike others who received radiotherapy before chemotherapy. In addition, the 2 groups were different in terms of comorbidity index which makes the comparison challenging. Moreover, the short median follow-up period for the TNT group is due to the novelty of this modality which has only been used recently.

In conclusion, our data show that TNT is superior to CRT in chemotherapy compliance. In addition, the pCR and tumor downstaging rates in the TNT group were numerically higher than the CRT group. No difference was noted in the 2-year DFS rates between the two treatment modalities. Finally, more studies are needed to truly compare the disease-free and OS rates between the two treatment modalities. 


\section{Acknowledgements}

This abstract was presented at the European Society of Medical Oncology ESMO 22nd World Congress on Gastrointestinal Cancer, which was held virtually between July 1st and 4th, 2020, and was published as Abstract no. P-134 in Annals of Oncology.

\section{Funding}

No funding was received.

\section{Availability of data and materials}

The datasets generated and/or analyzed during the current study are not publicly available due to the IRB restrictions but are available from the corresponding author on reasonable request.

\section{Authors' contributions}

AS conceived and designed the study. MAD and MC provided administrative support. AS provided study materials or patients. ZEH, YH, YB and MK collected and assembled data. ZEH, YH, YB, MK, DM, ST, MAD, MC and AS analyzed and interpreted data. All authors wrote the manuscript. AS and MC confirm the authenticity of all the raw data. All authors read and approved the final manuscript.

\section{Ethics approval and consent to participate}

The study was conducted in accordance with the Declaration of Helsinki (as revised in 2013). The study was approved via expedited review by the Biomedical Institutional Review Board Committee of the American University of Beirut Medical Center (IRB ID, BIO-2019-0024; Beirut, Lebanon). In this retrospective study, the IRB previously granted a waiver of the requirement to obtain informed consent.

\section{Patient consent for publication}

Not applicable.

\section{Competing interests}

The authors declare that they have no competing interests.

\section{References}

1. Sauer R, Becker H, Hohenberger W, Rödel C, Wittekind C Fietkau R, Martus P, Tschmelitsch J, Hager E, Hess CF, et al: Preoperative vs. postoperative chemoradiotherapy for rectal cancer. N Engl J Med 351: 1731-1740, 2004.

2. Ruo L and Guillem JG: Major 20th-century advancements in the management of rectal cancer. Dis Colon Rectum 42: 563-578, 1999.

3. Maas M, Nelemans PJ, Valentini V, Crane CH, Capirci C, RödelC, Nash GM, Kuo LJ, Glynne-Jones R, García-Aguilar J, et al: Adjuvant chemotherapy in rectal cancer: Defining subgroups who may benefit after neoadjuvant chemoradiation and resection: A pooled analysis of 3,313 patients. Int J Cancer 137: 212-220, 2015.

4. Biagi JJ, Raphael MJ, Mackillop WJ, Kong W, King WD and Booth CM: Association between time to initiation of adjuvant chemotherapy and survival in colorectal cancer: A systematic review and meta-analysis. JAMA 305: 2335-2342, 2011.
5. Breugom AJ, Swets M, Bosset JF, Collette L, Sainato A, Cionini L, Glynne-Jones R, Counsell N, Bastiaannet E, van den Broek CB, et al: Adjuvant chemotherapy after preoperative (chemo)radiotherapy and surgery for patients with rectal cancer: A systematic review and meta-analysis of individual patient data. Lancet Oncol 16: 200-207, 2015.

6. Bujko K, Glimelius B, Valentini V, Michalski W and Spalek M: Postoperative chemotherapy in patients with rectal cancer receiving preoperative radio(chemo)therapy: A meta-analysis of randomized trials comparing surgery \pm a fluoropyrimidine and surgery + a fluoropyrimidine \pm oxaliplatin. Eur J Surg Oncol 41: 713-723, 2015.

7. van Gijn W, Marijnen CA, Nagtegaal ID, Kranenbarg EM, Putter H, Wiggers T, Rutten HJ, Påhlman L, Glimelius B and van de Velde CJ; Dutch Colorectal Cancer Group: Preoperative radiotherapy combined with total mesorectal excision for resectable rectal cancer: 12-year follow-up of the multicentre, randomised controlled TME trial. Lancet Oncol 12: 575-582, 2011.

8. Kapiteijn E, Marijnen CA, Nagtegaal ID, Putter H, Steup WH, Wiggers T, Rutten HJ, Pahlman L, Glimelius B, van Krieken $\mathrm{JH}$, et al: Preoperative radiotherapy combined with total mesorectal excision for resectable rectal cancer. N Engl J Med 345: 638-646, 2001.

9. Hartley A, Ho KF, McConkey C and Geh JI: Pathological complete response following pre-operative chemoradiotherapy in rectal cancer: Analysis of phase II/III trials. Br J Radiol 78: 934-938, 2005.

10. Cotte E, Passot G, Decullier E, Maurice C, Glehen O, François Y, Lorchel F, Chapet O and Gerard JP: Pathologic response, when increased by longer interval, is a marker but not the cause of good prognosis in rectal cancer: 17-year follow-up of the Lyon R90-01 randomized trial. Int J Radiat Oncol Biol Phys 94: 544-553, 2016.

11. Park IJ, You YN, Agarwal A, Skibber JM, Rodriguez-Bigas MA, Eng C, Feig BW, Das P, Krishnan S, Crane CH, et al: Neoadjuvant treatment response as an early response indicator for patients with rectal cancer. J Clin Oncol 30: 1770-1776, 2012.

12. Pretzsch E, Bösch F, Neumann J, Ganschow P, Bazhin A, Guba M, Werner J and Angele M: Mechanisms of metastasis in colorectal cancer and metastatic organotropism: Hematogenous vs. peritoneal spread. J Oncol 2019: 7407190, 2019.

13. Sauer R, Liersch T, Merkel S, Fietkau R, Hohenberger W, Hess C, Becker H, Raab HR, Villanueva MT, Witzigmann H, et al: Preoperative vs. postoperative chemoradiotherapy for locally advanced rectal cancer: Results of the German CAO/ARO/AIO-94 randomized phase III trial after a median follow-up of 11 years. J Clin Oncol 30: 1926-1933, 2012.

14. Hospers G, Bahadoer RR, Dijkstra EA, Etten BV, Marijnen C, Putter H, Meershoek-Klein Kranenbarg E, Roodvoets AG, Nagtegaal ID, Regina GH Beets-Tan, et al: Short-course radiotherapy followed by chemotherapy before TME in locally advanced rectal cancer: The randomized RAPIDO trial. J Clin Oncol 38 (Suppl 15): S4006, 2020.

15. Bahadoer RR, Dijkstra EA, van Etten B, Marijnen CAM, Putter H, Kranenbarg EM, Roodvoets AGH, Nagtegaal ID, Beets-Tan RGH, Blomqvist LK, et al: Short-course radiotherapy followed by chemotherapy before total mesorectal excision (TME) vs. preoperative chemoradiotherapy, TME, and optional adjuvant chemotherapy in locally advanced rectal cancer (RAPIDO): A randomised, open-label, phase 3 trial. Lancet Oncol 22: 29-42, 2021.

16. Becker K, Mueller JD, Schulmacher C, Ott K, Fink U, Busch R, Böttcher K, Siewert JR and Höfler H: Histomorphology and grading of regression in gastric carcinoma treated with neoadjuvant chemotherapy. Cancer 98: 1521-1530, 2003.

17. Delibegovic S: Introduction to total mesorectal excision. Med Arch 71: 434-438, 2017.

18. Bosset JF, Calais G, Mineur L, Maingon P, Stojanovic-Rundic S, Bensadoun RJ, Bardet E, Beny A, Ollier JC, Bolla M, et al: Fluorouracil-based adjuvant chemotherapy after preoperative chemoradiotherapy in rectal cancer: Long-term results of the EORTC 22921 randomised study. Lancet Oncol 15: 184-190, 2014.

19. Sainato A, Cernusco Luna Nunzia V, Valentini V, De Paoli A, Maurizi ER, Lupattelli M, Aristei C, Vidali C, Conti M, Galardi A, et al: No benefit of adjuvant Fluorouracil Leucovorin chemotherapy after neoadjuvant chemoradiotherapy in locally advanced cancer of the rectum (LARC): Long term results of a randomized trial (I-CNR-RT). Radiother Oncol 113: 223-229, 2014. 
20. Schmoll HJ, Haustermans K, Price TJ, Nordlinger B, Hofheinz R, Daisne JF, Janssens J, Brenner B, Schmidt P, Reinel H, et al: Preoperative chemoradiotherapy and postoperative chemotherapy with capecitabine +/-oxaliplatin in locally advanced rectal cancer: Final results of PETACC-6. J Clin Oncol 36 (Suppl 15): S3500, 2018

21. Rödel C, Graeven U, Fietkau R, Hohenberger W, Hothorn T, Arnold D, Hofheinz RD, Ghadimi M, Wolff HA, Lang-Welzenbach M, et al: Oxaliplatin added to fluorouracil-based preoperative chemoradiotherapy and postoperative chemotherapy of locally advanced rectal cancer (the German CAO/ARO/AIO-04 study): Final results of the multicentre, open-label, randomised, phase 3 trial. Lancet Oncol 16: 979-989, 2015.

22. Mari GM, Maggioni D, Crippa J, Costanzi ATM, Scotti MA, Giardini V, Garancini M, Cocozza E, Borroni G, Benzoni I, et al: Compliance to adjuvant chemotherapy of patients who underwent surgery for rectal cancer: Report from a Multi-institutional Research Network. World J Surg 43: 2544-2551, 2019.

23. Marco MR, Zhou L, Patil S, Marcet JE, Varma MG, Oommen S, Cataldo PA, Hunt SR, Kumar A, Herzig DO, et al: Consolidation mFOLFOX6 chemotherapy after chemoradiotherapy improves survival in patients with locally advanced rectal cancer: Final results of a multicenter phase II trial. Dis Colon Rectum 61: $1146-1155,2018$

24. Garcia-Aguilar J, Chow OS, Smith DD, Marcet JE, Cataldo PA, Varma MG, Kumar AS, Oommen S, Coutsoftides $T$, Hunt SR, et al: Effect of adding mFOLFOX6 after neoadjuvant chemoradiation in locally advanced rectal cancer: A multicentre, phase 2 trial. Lancet Oncol 16: 957-966, 2015.

25. Ngan SY, Burmeister B, Fisher RJ, Solomon M, Goldstein D, Joseph D, Ackland SP, Schache D, McClure B, McLachlan SA, et al: Randomized trial of short-course radiotherapy vs. long-course chemoradiation comparing rates of local recurrence in patients with T3 rectal cancer: Trans-Tasman Radiation Oncology Group trial 01.04. J Clin Oncol 30: 3827-3833, 2012.

26. Erlandsson J, Holm T, Pettersson D, Berglund Å, Cedermark B, Radu C, Johansson H, Machado M, Hjern F, Hallböök O, et al: Optimal fractionation of preoperative radiotherapy and timing to surgery for rectal cancer (Stockholm III): A multicentre, randomised, non-blinded, phase 3, non-inferiority trial. Lancet Oncol 18: 336-346, 2017.

27. Ciseł B, Pietrzak L, Michalski W, Wyrwicz L, Rutkowski A, Kosakowska E, Cencelewicz A, Spałek M, Polkowski W, Jankiewicz M, et al: Long-course preoperative chemoradiation vs. 5x5 Gy and consolidation chemotherapy for clinical T4 and fixed clinical T3 rectal cancer: Long-term results of the randomized Polish II study. Ann Oncol 30: 1298-1303, 2019.
28. Fokas E, Allgäuer M, Polat B, Klautke G, Grabenbauer GG, Fietkau R, Kuhnt T, Staib L, Brunner T, Grosu AL, et al: Randomized phase II trial of chemoradiotherapy plus induction or consolidation chemotherapy as total neoadjuvant therapy for locally advanced rectal cancer: CAO/ARO/AIO-12. J Clin Oncol 37: 3212-3222, 2019.

29. Manthravadi S, Sun W, Saeed A, Baranda JC and Kasi A: Total neoadjuvant therapy compared with standard therapy in locally advanced rectal cancer: A systematic review and meta-analysis. J Clin Oncol 37 (Suppl 4): S709, 2019.

30. Chapman W Jr, Roxburgh C, Makhdoom B, Roy A, Youssef FF, Brady P, Olsen J, Kim H, Pedersen K, Mutch H, et al: Rectal cancer downstaging is significantly improved with different regimens of total neoadjuvant therapy. Int J Radiation Oncol Biol Phys 102 (Suppl): S65-S66, 2018.

31. Garcia-Aguilar J, Patil S, Kim JK, Yuval JB, Thompson H, Verheij F, Lee M and Saltz LB; on behalf of the OPRA Consortium: Preliminary results of the organ preservation of rectal adenocarcinoma (OPRA) trial. J Clin Oncol 38 (Suppl 15): S4008, 2020.

32. Shui L, Yang X, Li J, Yi C, Sun Q and Zhu H: Gut microbiome as a potential factor for modulating resistance to cancer immunotherapy. Front Immunol 10: 2989, 2020.

33. Zhang MX, Li XB, Guan BJ, Guan GX, Lin XY, Wu XD, $\mathrm{Chi} \mathrm{P}$ and $\mathrm{Xu} \mathrm{BH}$ : Dose escalation of preoperative short-course radiotherapy followed by neoadjuvant chemotherapy in locally advanced rectal cancer: Protocol for an open-label, single-centre, phase I clinical trial. BMJ Open 9: e025944, 2019.

34. Yuki S, Bando H, Tsukada Y, Inamori K, Komatsu Y, Homma S, Uemura M, Kato T, Kotani D, Fukuoka S, et al: Short-term results of VOLTAGE-A: Nivolumab monotherapy and subsequent radical surgery following preoperative chemoradiotherapy in patients with microsatellite stable and microsatellite instability-high locally advanced rectal cancer. J Clin Oncol 38 (Suppl 15): 4100, 2020.

35. Capdevila J, Declara IM, Martinez MCR, Maurel J, Hernando J, Alonso V, Graña Suárez B, Plazas JG, Losa F, Vera R, et al: Phase II study of durvalumab plus total neoadjuvant therapy (TNT) in locally advanced rectal cancer: The GEMCAD-1703 DUREC trial. J Clin Oncol: 38 (Suppl 15): TPS4122, 2020.

36. Shamseddine A, Zeidan Y, Khalifeh IM, Kattan JG, Turfa R, Mukherji D, Naji Temraz S, Jamali, F, Hani Shaib Y, Soweid A, et al: Short-course radiation followed by mFOLFOX-6 plus avelumab for locally advanced rectal adenocarcinoma. J Clin Oncol 38 (Suppl 4): S139, 2020. 